\title{
Dependência de smartphone em adolescentes, parte 1: revisão de escopo
}

\section{La dependencia de smartphone en adolescentes, parte 1: revisión del alcance Smartphone fiddiction in Adolescents, Part 1: A Scoping Review}

\author{
Bruna Hinnah Borges Martins de Freitas \\ Maria Aparecida Munhoz Gaíva \\ Fabiane Blanco Silva Bernardino \\ Universidade Federal de Mato Grosso \\ Paula Manuela Jorge Diogo \\ Escola Superior de Enfermagem de Lisboa
}

Doi: https://doi.org/10.12804/revistas.urosario.edu.co/apl/a.8404

\begin{abstract}
Resumo
Face a presença constante dos smartphones na vida dos adolescentes e ao aumento do tempo de tela observado nos últimos anos, este estudo objetivou mapear as evidências disponíveis sobre a dependência de smartphone em adolescentes. Trata-se da primeira parte de uma revisão de escopo. Um total de 67 artigos foram publicados entre 2009 e 2019, com um incremento de $31,74 \%$ ao ano. A maioria das pesquisas $(N=28$; $41,79 \%$ ), foram desenvolvidas na Coreia do Sul, pela área de psicologia $(N=21 ; 31,34 \%)$ e pelo método transversal $(N=49 ; 73,13 \%) .14$ escalas autoaplicáveis foram identificadas para o uso com adolescentes, sendo que, as mais utilizadas foram a Smartphone Addiction
\end{abstract}

Proneness Scale e a Smartphone Addiction Scale- Short Version. Grande parte das escalas contempla os domínios: abstinência, tolerância, compulsão e efeito da dependência na vida diária. Conclui-se que a dependência de smartphone é um problema de saúde pública em ascensão no mundo e pode comprometer a saúde dos adolescentes. Tal construto tem sido mensurado por escalas validadas e confiáveis, porém, não foram identificados estudos brasileiros no período investigado. Portanto, faz-se necessário adaptar e validar instrumentos com esta população para melhor compreensão do fenômeno no Brasil.

Palavras-chave: dependência do smartphone; comportamento do adolescente; saúde do adolescente.

Bruna Hinnah Borges Martins de Freitas ORCID ID: https://orcid.org/0000-0002-6652-593X

Maria Aparecida Munhoz Gaíva ORCID ID: https://orcid.org/0000-0002-8666-9738

Fabiane Blanco Silva Bernardino ORCID ID: https://orcid.org/0000-0003-0339-9451

Paula Manuela Jorge Diogo ORCID ID: https://orcid.org/0000-0003-4828-3452

Dirigir correspondência à Bruna Hinnah Borges Martins de Freitas. Endereço: Faculdade de Enfermagem, Universidade Federal de Mato Grosso, Av. Fernando Corrêa da Costa n²367, Bairro Boa Esperança, Cuiabá, Mato Grosso, 78060-900, Brasil. Correio eletrônico: bruna.freitas@ufmt.br

Para citar este artigo: Freitas, B. H. B. M., Gaíva, M. A. M., Bernardino, F. B. S., \& Diogo, P. M. J. (2021). Dependência de smartphone em adolescentes, parte 1: revisão de escopo. Avances en Psicología Latinoamericana, 39(2), 1-17. https://doi.org/10.12804/revistas. urosario.edu.co/apl/a.8404 


\section{Resumen}

Dada la presencia constante de los teléfonos inteligentes en la vida de los adolescentes y el aumento del tiempo de pantalla observado en los últimos años, el presente estudio tuvo como objetivo mapear la evidencia disponible sobre la dependencia del smartphone en adolescentes. Esta es una revisión del alcance. Se publicaron un total de 67 artículos entre 2009 y 2019, con un aumento del $31.74 \%$ por año. La mayor parte de la investigación se realizó en Corea del Sur $(N=28$; $41,79 \%)$, por el área de psicología $(N=21 ; 31,34 \%)$ y por el método transversal $(N=49 ; 73,13 \%)$. Se identificaron catorce escalas autoaplicables para su uso con adolescentes, y las más utilizadas fueron la Smartphone Addiction Proneness Scale y la Smartphone Addiction Scale-Short Version. La mayoría de las escalas incluyen los siguientes dominios: abstinencia, tolerancia, compulsión y el efecto de la dependencia en la vida diaria. Se concluye que la dependencia de los smartphones es un problema de salud pública creciente en el mundo y puede comprometer la salud de los adolescentes. Esta construcción se ha medido mediante escalas validadas y confiables, pero no se han identificado estudios brasileños en el periodo investigado, por lo que es necesario adaptar y validar los instrumentos con esta población, para comprender mejor el fenómeno en Brasil.

Palabras clave: adicción a los teléfonos inteligentes; comportamiento adolescente; salud del adolescente.

\section{fibstract}

Given the constant presence of smartphones in adolescents' lives and the increase in screen time observed in recent years, this study aimed to map the evidence available on adolescents' smartphone addiction. This is the first part of a scoping review. A total of 67 articles were published between 2009 and 2019, with an increase of $31.74 \%$ per year. Most of the research was conducted in South Korea $(\mathrm{N}=28 ; 41.79 \%)$, in psychology $(\mathrm{N}=21$; $31.34 \%)$ and by the cross-sectional method $(\mathrm{N}=49$; $73.13 \%$ ). Fourteen self-applicable scales were identified for use with adolescents, the most used being the Smartphone Addiction Proneness Scale and the Smartphone Addiction Scale-Short Version. Most scales include the following domains: abstinence, tolerance, compulsion, and the effect of addiction on daily life. It was concluded that smartphone addiction is a growing public health problem on a global scale that may compromise adolescents' health. This construct has been measured by validated and reliable scales, but no Brazilian studies have been identified in the period investigated, so it is necessary to adapt and validate instruments with this population to better understand the phenomenon in Brazil.

Keywords: Smartphone addiction; adolescent behavior; adolescent health.

Considera-se o smartphone um símbolo contemporâneo de inovação das Tecnologias Digitais de Informação e Comunicação (TDIC), que assegura a conectividade com mobilidade aos usuários de maneira versátil e multifuncional (Duke \& Montag, 2017). Ao final de 2014, mais de $93 \%$ da população mundial os possuía (Birenboim \& Shoval, 2016). Ainda, há previsão de que o número de dispositivos conectados serão mais de três vezes a população global até 2023. Portanto, haverá 3,6 dispositivos em rede per capita até 2023, acima dos 2,4 dispositivos em rede per capita em 2018 (CISCO, 2020).

No Brasil, constatou-se, na última Pesquisa Nacional por Amostra de Domicílios Contínua (PNAD) no final de 2018, que $98.1 \%$ dos brasileiros acessam a internet por meio do telefone celular (Instituto Brasileiro de Geografia e Estatística [IBGE], 2020). A onipresença do dispositivo parece capaz de alterar as rotinas diárias, hábitos, comportamentos sociais, valores emancipativos, relações familiares e de trabalho, e interações sociais (Birenboim \& Shoval, 2016; Duke \& Montag, 2017) e, embora eles possam melhorar muitos aspectos da vida humana, o seu uso excessivo pode levar a dependência (Khoury et al., 2017).

A dependência de smartphone (DS) é considerada um vício tecnológico, ou seja, vício comportamental não químico, envolvendo interação entre o homem e a máquina (Griffiths, 1996). É um conceito ainda em construção, descrito por alguns 
autores como nomofobia, que significa o pânico de estar afastado do aparelho (Jeong et al., 2016), caracterizado pelo uso excessivo, incontrolável e prejudicial do mesmo, negligenciando outras áreas da vida (Lin et al., 2014).

O uso do termo "adição ao smartphone" é comum em vários países para descrever problemas graves relacionados ao uso compulsivo do aparelho, mas o vocábulo "adição" é omitido da terminologia diagnóstica do Manual Diagnóstico e Estatístico de Transtornos Mentais (DSM-5), em razão de sua definição vaga e conotação potencialmente negativa. Adição a não substância é considerada um transtorno comportamental (também denominado adição comportamental), que apesar de não estar relacionado ao abuso de substância, compartilha algumas características com a adição induzida por ela, contudo, não tem sido aplicado como termo diagnóstico, sendo comumente usada em contextos não médicos (American Psychiatric Association [APA], 2014).

Ainda, há na literatura um uso quase indistinguível ou pouco diferenciado dos termos "uso problemático", "abuso" e "adição/dependência" (Gutiérrez et al., 2016). Considerando ainda que a dependência é um fenômeno caracterizado por tolerância, sintomas de abstinência, comportamento compulsivo, estreitamento de repertório e problemas sociais (Khoury et al., 2017), os aspectos supracitados sugerem que se pode assumir o termo "dependência de smartphone".

Os adolescentes, como um segmento social mais susceptível às transformações das tecnologias digitais, têm maior propensão de desenvolver a dependência (Silva \& Silva, 2017). Dentre as principais características que fazem os adolescentes se sentirem atraídos pelo aparelho, estão: autonomia, identidade e prestígio, fascínio pelas aplicações tecnológicas, possibilidade de atividade de lazer e estabelecimento de relações interpessoais (Ruiz et al., 2016).

Considerando as características neuropsicobiológicas (Telzer, 2016) e vulnerabilidade ao uso das tecnologias por esse grupo populacional (Silva \&
Silva, 2017), se faz necessário a implementação de instrumentos válidos e confiáveis que mensurem a dependência autorreferida de smartphone em adolescentes. Instrumentos de medida são partes integrantes da prática clínica, da avaliação em saúde e de pesquisas. Estes só são úteis e capazes de apresentar resultados cientificamente comprovados quando são desenvolvidos de maneira apropriada e apresentam boas qualidades psicométricas (Coluci et al., 2015).

Neste sentido, torna-se necessário rastrear tal fenômeno durante a vigilância em saúde realizada por enfermeiros e demais profissionais no âmbito do cuidado primário em saúde. Por meio deste, é possível detectar situações de maior vulnerabilidade entre os adolescentes e, então, planejar e implementar medidas de prevenção deste comportamento de risco e de promoção de comportamentos adaptativos a fim de garantir o desenvolvimento pleno desse grupo populacional.

Considerando os aspectos supracitados, o objetivo da presente revisão foi mapear as evidências disponíveis sobre a dependência de smartphone em adolescentes.

\section{Método}

Trata-se de uma revisão de escopo, a qual permite revisar de forma mais abrangente determinada temática e sintetizar as evidências disponíveis, mapeando a literatura existente em determinado campo de interesse e identificando lacunas na base do conhecimento pesquisada (Peters et al., 2020).

Foram seguidas as etapas previstas para o desenvolvimento deste tipo de estudo: (1) definição e alinhamento do objetivo e questão de pesquisa; (2) definição dos critérios de inclusão; (3) descrição da abordagem planejada para busca de evidências, seleção, extração de dados e apresentação de evidências; (4) busca de evidências; (5) seleção das evidências; (6) extração das evidências; (7) análise das evidências; (8) apresentação dos resultados e 
(9) resumo das evidências em relação ao objetivo da revisão, refletindo sobre as conclusões e anotando as implicações dos resultados (Peters et al., 2020).

A pergunta de pesquisa foi elaborada utilizando a estratégia PCC, que preconiza como elementos fundamentais o mnemônico: P - População; C - Conceito e C-Contexto (Peters et al., 2020). Foram definidos os elementos: P (adolescentes); C (dependência de smartphone) e C (tecnologia). A partir dessa definição, foi elaborada a seguinte pergunta de pesquisa: "Quais as evidências disponíveis sobre a dependência de smartphone em adolescentes?".

As bases de dados pesquisadas foram: National Library of Medicine (MEDLINE/PubMed), Current Nursing and Allied Health Literature (CINAHL), scopus, Web of Science, Literatura Latino-americana e do Caribe em Ciências da Saúde (LILACS) e American Psychological Association (PsycINFO). Essas bases foram selecionadas por serem as principais na área da saúde e terem acesso via Comunidade Acadêmica Federada (CAFe) do Portal de Periódicos da Coordenação de Aperfeiçoamento de Pessoal de Nível Superior (CAPES).

Em seguida, para assegurar uma busca criteriosa, definiram-se os descritores controlados [Descritores em Ciência da Saúde (DeCS), Medical Subject Headings (MesH) e CINAHL Headings] e não controlados (palavras-chave). Estes foram sintetizados conforme a estratégia PCC e suas combinações foram usadas para construir as estratégias: P: "Adolescents" OR "Adolescence" OR "Youth" OR "Teenagers" OR "Teen"; AND C: "smartphone addiction" OR "smartphone dependence" OR "mobile phone addiction" OR "mobile phone dependence". Pelo fato do C (contexto) tratar-se do contexto tecnológico, optou-se por não selecionar palavras-chave e termos correspondentes a ele especificamente, pois compreende-se que a palavra "smartphone" já remete a este contexto.

A busca nas bases foi realizada no período de 06 a 29 de maio de 2019, por dois revisores de forma independente, limitando-se aos artigos científicos originais com acesso aberto, nos idiomas inglês, espanhol ou português, sem delimitação temporal. Foram excluídos os artigos que não responderam à questão norteadora, os de revisão e as notas editoriais.

A figura 1 apresenta o diagrama de fluxo da seleção dos artigos, conforme a recomendação do Preferred Reporting Items for Systematic Reviews and Meta-Analyses (PRISMA) (Page et al., 2021). A lista de referências dos artigos incluídos, a partir das bases de dados, foi consultada a fim de identificar estudos adicionais que não haviam sido mapeados nas etapas anteriores.

Depois, os artigos foram mapeados mediante indicadores de coleta de dados digitados em planilhas eletrônicas disponíveis no programa Microsoft Excel 365®, designados por: ano de publicação, país de origem, título, autores, objetivo, população de estudo/amostra, desenho metodológico, instrumentos aplicados e resultados principais.

Por fim, na quinta etapa, buscando identificar as evidências disponíveis para responder à questão norteadora, os dados foram analisados e sintetizados a partir das seguintes categorias: (I) Caracterização das publicações; (II) Características definidoras da dependência de smartphone em adolescentes; (III) Instrumentos de avaliação da dependência de smartphone em adolescentes; (Iv) Prevalência e fatores associados a dependência de smartphone em adolescentes. Para a divulgação dos resultados, a revisão foi dividida em duas partes: parte 1 - categorias I, II e III; e parte 2 - categoria IV (Freitas et al., 2021). A fim de responder ao objetivo deste artigo foram utilizadas as categorias da parte 1 .

As evidências mapeadas foram resumidas, extraindo conclusões, observando implicações dos resultados e identificando as lacunas. Os resultados também foram analisados por estatística descritiva e com a análise de série temporal por meio da regressão de Prais Winsten (Antunes \& Cardoso, 2015). 


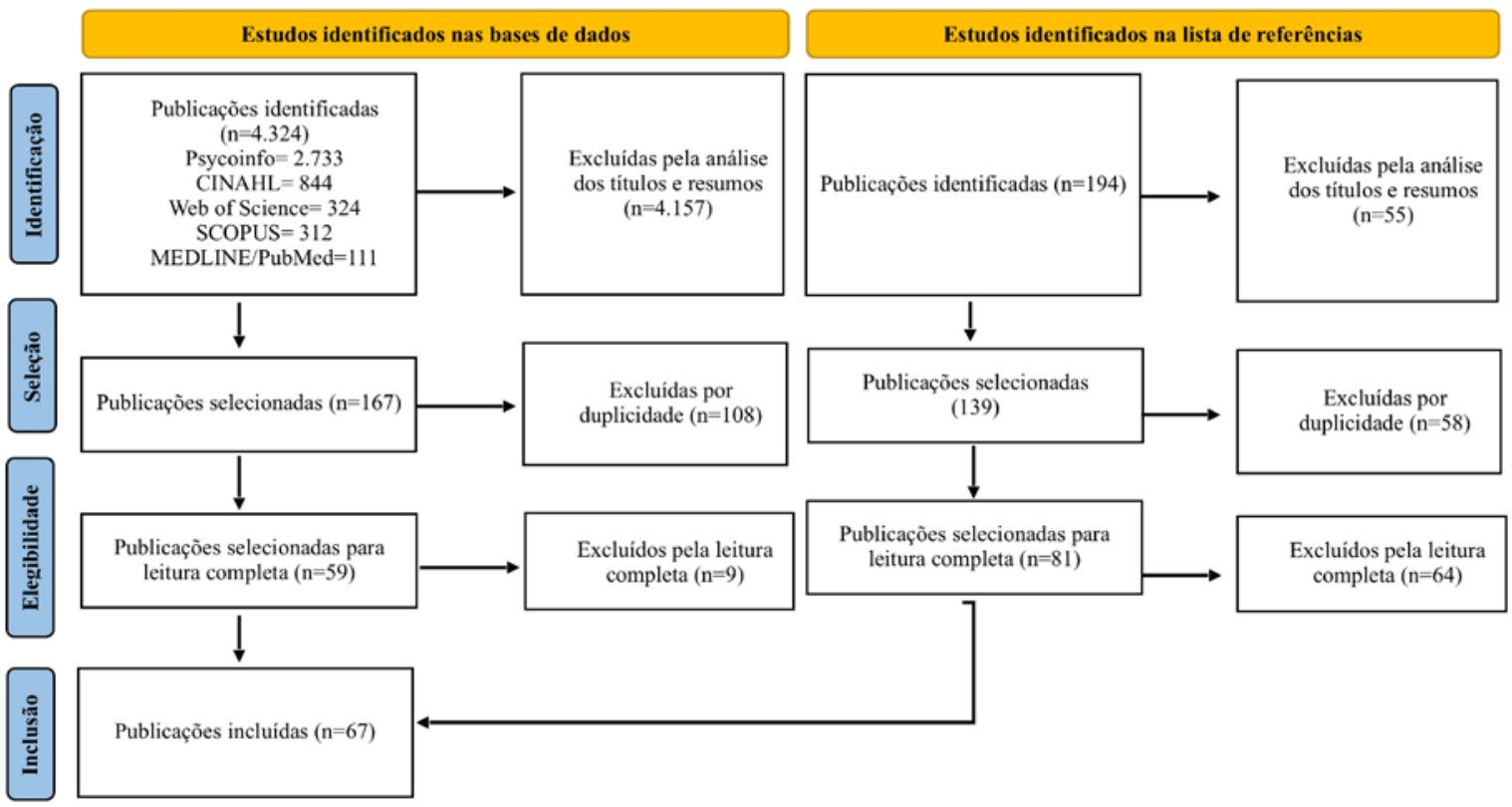

Figura 1. Diagrama de fluxo da seleção dos artigos. Cuiabá, Brasil, 2021

Fuente: adaptado do PRISMA (Page et al., 2021).

\section{Resultados}

\section{Caracterização das publicações}

As 67 publicações incluídas foram divulgadas entre 2009 e 2019, como exposto na figura 2. Veri- fica-se tendência ascendente das publicações sobre dependência de smartphone em adolescentes no período, com uma variação percentual anual de $31,74 \%(p<0.05)$. Observa-se maior incremento de produções a partir de 2014, com maior frequência no ano de 2018 ( $N=20 ; 29,85 \%)$.

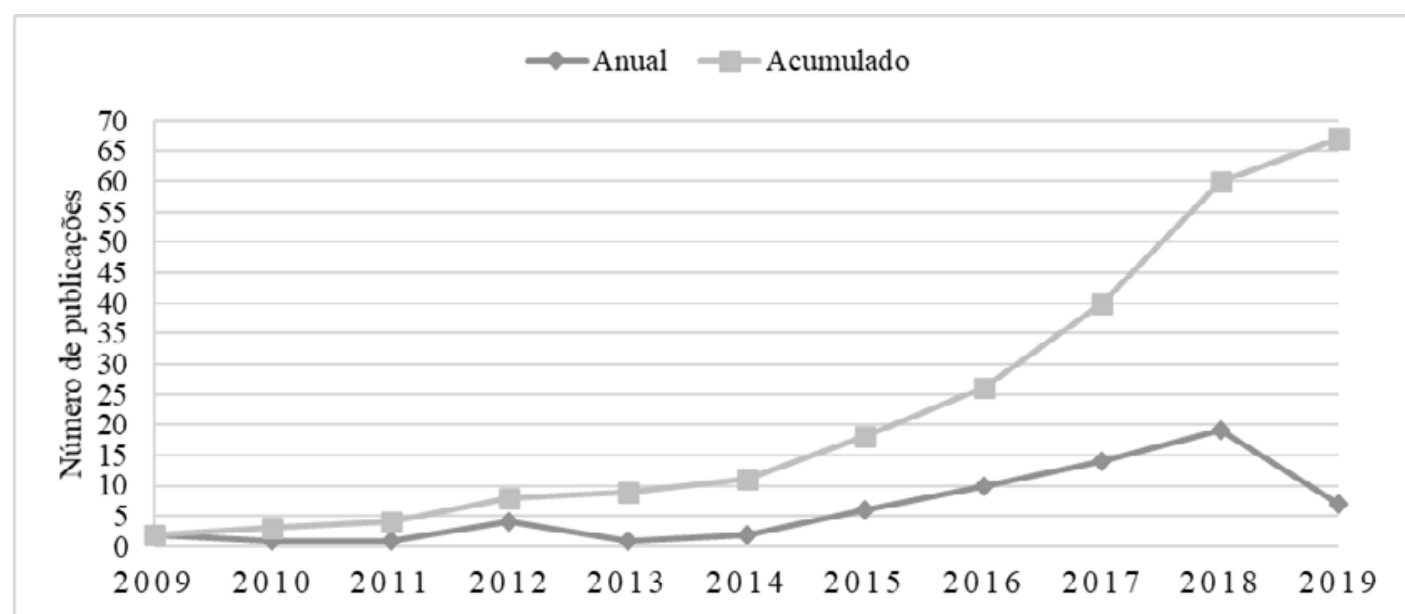

Figura 2. Produção científica sobre dependência de smartphone em adolescentes de 2009 até maio de 2019. Cuiabá, Brasil, 2021 
Grande parte das pesquisas foi desenvolvida na Coreia do $\mathrm{Sul}(N=28 ; 41,79 \%)$, conforme pode ser visualizado na figura 3 . Destaca-se que não foram identificados por meio desta revisão estudos desenvolvidos em países da América, como nos Estados Unidos e Brasil, por exemplo. A principal área que investigou o fenômeno foi a
Psicologia $(N=21 ; 31,34 \%)$, seguida pela Medicina $(N=16 ; 23,88 \%)$, Educação $(N=8 ; 11,94 \%)$, Enfermagem $(N=6 ; 8,96 \%)$, Saúde Pública $(N=5$; $7,46 \%)$, Comunicação ( $N=3 ; 4,48 \%)$, Educação Física e Serviço Social ( $N=2 ; 2,99 \%$ cada); Sociologia, Administração, Economia e Nutrição $(N=1 ; 1,49 \%$ cada $)$.

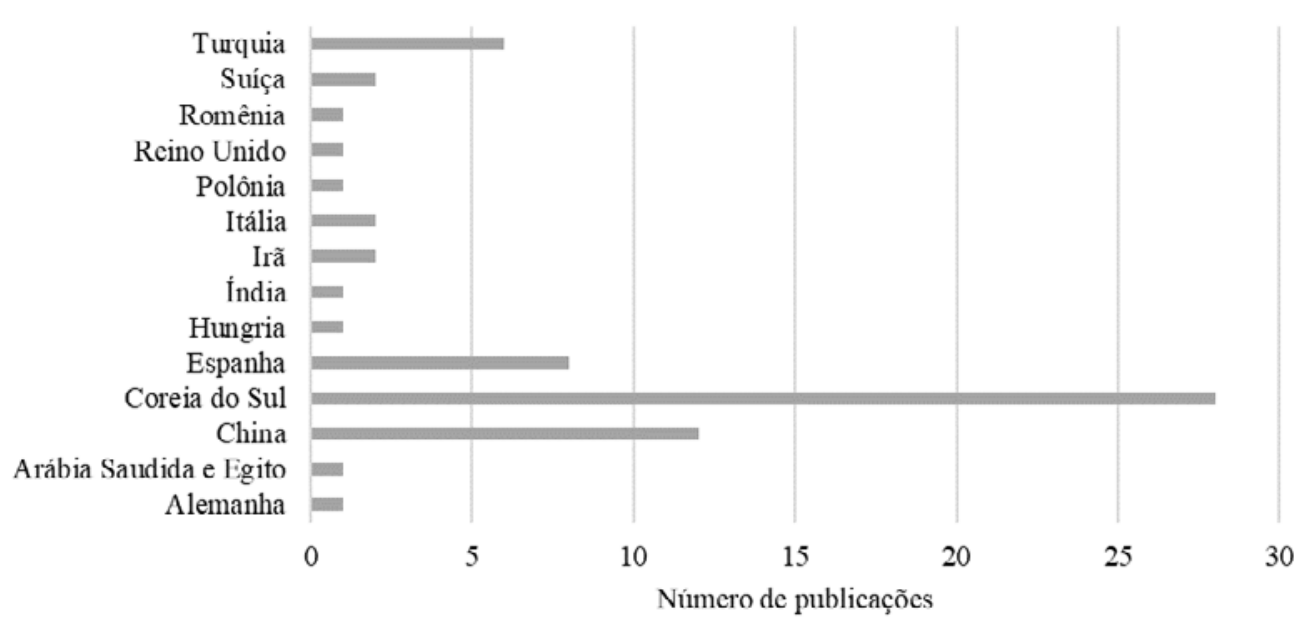

Figura 3. Produção científica sobre dependência de smartphone em adolescentes por país. Cuiabá, Brasil, 2021

A maioria dos estudos foi do tipo transversal $(N=49 ; 73,13 \%)$, seguido pelos estudos metodológicos $(N=12 ; 17,91 \%)$; estudo de intervenção quase-experimental $(N=2 ; 2,99 \%)$ e; caso-controle, coorte, método misto e estudo de caso $(N=1 ; 1,49 \%$ cada). Ressalta-se que alguns estudos transversais também validaram instrumentos para alcançarem seus objetivos.

\section{Características definidoras da dependência de smartphone em adolescentes}

Para alguns autores, a DS compartilha características previstas pelo Manual Diagnóstico e Estatístico de Transtornos Mentais IV e V (APA, 2014) para abuso de substâncias e jogo patológico, como aumento da tolerância, abstinência, efeitos negativos, redução de atividades, perda de controle, fuga dos problemas e desejo de estar conectado (Beranuy et al., 2009). Pesquisadores concordam que os critérios da dependência de smartphone se assemelham aos critérios do transtorno de jogos, exceto pela característica “perseguir perdas”, específica dos jogadores (Körmendi et al., 2016).

Ao comparar os sintomas da DS com as concepções de vício tecnológico de Griffiths (2000), foi possível identificar a presença dos seguintes componentes: preocupação, modificação de humor, tolerância, abstinência e recaída (Körmendi et al., 2016).

Concomitantemente, ao compará-lo com os critérios de vício comportamental de Goodman (1990), os autores encontraram características semelhantes, como por exemplo: falha recorrente em resistir a impulsos para o comportamento 
especificado, aumento da sensação de tensão imediatamente antes de iniciá-lo, prazer ou alívio neste momento, falta de controle, preocupação e envolvimento em maior medida ou por um período mais longo do que o pretendido; manutenção do comportamento quando esperado cumprir obrigações ocupacionais, acadêmicas, domésticas ou sociais; tolerância, inquietação ou irritabilidade e persistência nos sintomas da perturbação por pelo menos um mês ou durante um período mais longo (Körmendi et al., 2016).

Pesquisa desenvolvida na Coreia do Sul mostrou que os adolescentes com DS apresentaram perturbação da função adaptativa, abstinência e tolerância, com aumento na concentração de cortisol (Chun et al., 2018). Encontrou-se menor conectividade funcional entre o córtex orbitofrontal (COF) direito e o núcleo accumbens (NAcc), entre o COF esquerdo e córtex cingulado médio (CCM) e, maior conectividade entre o CCM e o NAcc em adolescentes com uso excessivo de smartphone. A correlação entre a conectividade do COF direito com o NAcc e os sintomas de abstinência e concentração de cortisol foi negativa. A conectividade funcional entre o COF e o NAcc e entre o COF e o CCM está relacionada ao controle cognitivo de estímulos emocionais, incluindo recompensa. Portanto, esses resultados sugerem que a DS parece estar relacionada a conectividade funcional alterada entre regiões vinculadas ao controle cognitivo e sistema de recompensas (Chun et al., 2018).

A tolerância e abstinência foram reconhecidas como características da DS por outros autores (Foerster et al., 2015; Güzeller \& Coşguner, 2012; Kim et al., 2014; Kwon et al., 2013a; Yen et al., 2009), assim como perturbação da função adaptativa (Kim et al., 2014; Kwon et al., 2013a). Há relato de fuga dos problemas, craving (Foerster et al., 2015; Güzeller \& Coşguner, 2012) e antecipação positiva nesses indivíduos (Kwon et al., 2013a). Ademais, o medo de perder o aparelho é reconhecido como uma das principais características dos dependentes de smartphone (Cocoradă et al., 2018; Santana-Vega et al., 2019).

\section{Instrumentos de avaliação da dependência de smartphone em adolescentes}

A compreensão de como as pessoas usam o smartphone é importante, principalmente para medir o impacto que isso pode ocasionar nos indivíduos e sociedade. No entanto, esta depende predominantemente de avaliações autorreferidas até o momento. Foram identificadas nesta revisão 14 escalas validadas para o uso com adolescentes e estão dispostas na tabela 1. Dessas, as mais utilizadas para mensurar a DS pelos 67 artigos analisados foram a SAPS e a SAS-SV.

Tabela 1.

Instrumentos de avaliação da dependência de smartphone em adolescentes e fonte de dados. Cuiabá, MT, Brasil, 2019

\begin{tabular}{|c|c|c|}
\hline Instrumento & País & Autores do artigo (ano) \\
\hline $\begin{array}{l}\text { Cuestionario de Experiencias Relacionadas con el Móvil } \\
\text { (CERM) }\end{array}$ & Espanha & (Beranuy Fargues et al., 2009) \\
\hline $\begin{array}{l}\text { Problematic Cellular Phone Use Questionnaire } \\
\text { (PCPU-Q) }\end{array}$ & China & Yen et al. (2009) \\
\hline Problematic Mobile Phone Use Scale (PMPUS) & Turquia & Güzeller \& Coşguner (2012) \\
\hline Test of Mobile Phone Dependence (TMD) & Espanha & Chóliz (2012) \\
\hline $\begin{array}{l}\text { Mobile Phone Problem Use Scale para adolescente } \\
\text { (MPPUSA) }\end{array}$ & $\begin{array}{c}\text { Espanha } \\
\text { Reino Unido }\end{array}$ & $\begin{array}{l}\text { Lopez-Fernandez et al. (2012) } \\
\text { Lopez-Fernandez et al. (2014) }\end{array}$ \\
\hline
\end{tabular}




\begin{tabular}{lll}
\hline \multicolumn{1}{c}{ Instrumento } & País & Autores do artigo (ano) \\
\hline $\begin{array}{l}\text { Mobile Phone Problem Use Scale na versão curta e } \\
\text { (MPPUS-10) }\end{array}$ & Suíça & Foerster et al. (2015) \\
\hline $\begin{array}{l}\text { Smartphone Addiction Scale- Short Version (SAS-SV) } \\
\text { Smartphone Addiction Proneness Scale (SAPS) }\end{array}$ & Coreia do Sul & Kwon, Kim, et al. (2013) \\
\hline $\begin{array}{l}\text { Mobile Phone Involvement Questionnaire (MPIQ) } \\
\text { Mobile Phone Addiction Index (MPAI) }\end{array}$ & Coreia do Sul & Kim et al. (2014) \\
\hline $\begin{array}{l}\text { Mobile Phone Addiction Assessment Questionnaire } \\
\text { (KBUTK) }\end{array}$ & Espanha & Argumosa-Villar et al. (2017) \\
\hline $\begin{array}{l}\text { Nomophobia Questionnaire (NMP-Q) } \\
\text { Pobile Phone Dependence Questionnaire (MPDQ) }\end{array}$ & Piu et al. (2017) \\
\hline $\begin{array}{l}\text { Implicit Association Test measures for smartphone and } \\
\text { Internet addiction (IAT-SI). }\end{array}$ & Espanha & Warzecha \& Pawlak (2017) \\
\hline
\end{tabular}

Uma escala breve com 10 itens tipo Likert foi construída para avaliação do abuso de dispositivos móveis com base no instrumento Problemas Relacionados con el uso de Internet (PRI) (Blanco et al., 2002), denominada Cuestionario de Experiencias Relacionadas con el Móvil (CERM), que foi aplicada em uma amostra de 1.879 estudantes espanhóis. O primeiro fator (conflitos) apresentou uma consistência interna de 0,81 e o segundo (uso comunicacional e emocional) de 0,75 , com uma consistência interna geral de 0,80 (Beranuy et al., 2009).

O PCPU-Q foi desenvolvido com 12 itens, de acordo com as taxonomias da dependência de uso de substâncias do DSM-IV-TR para determinar a ocorrência de sintomas de DS em 10.191 adolescentes chineses. O alfa de Cronbach para os sete sintomas foi alto neste estudo $(0,85)$. Também foram recrutados 76 adolescentes e seus pais para um estudo piloto a fim de examinar a confiabilidade e a validade pelo coeficiente de concordância de Kappa. Os resultados indicaram que a confiabilidade teste-reteste dos itens foi satisfatório (Yen et al., 2009).

A PMPUS, por sua vez, foi desenvolvida e avaliada com 950 adolescentes turcos. A escala foi composta por três fatores: interferência com efeito negativo, compulsão/persistência e abstinência/ tolerância. Os resultados mostraram que os valores de confiabilidade dos itens e dos construtos apresentaram taxas satisfatórias em geral. Os valores de alfa de Cronbach variaram de 0,76 a 0,83 para os escores totais dos fatores baseados em consistência interna e escores totais gerais (Güzeller \& Coşguner, 2012).

Em 2012, foi desenvolvido o instrumento TMD e validado com 2.486 adolescentes espanhóis. Os 22 itens finais foram construídos de acordo com os critérios contidos no DSM-IV-TR para transtorno de dependência, no formato tipo Likert e composto pelos seguintes fatores: abstinência, falta de controle/problemas e tolerância/interferência. Essa escala apresentou alta consistência interna (alfa de Cronbach = 0,94) (Chóliz, 2012).

Neste mesmo ano, a MPPUs foi adaptada para a população adolescente (MPPUSA) (Lopez-Fernandez et al., 2012). A escala possui 27 itens em escala Likert de 10 pontos, contendo sintomas de dependência, como: tolerância, fuga dos problemas, abstinência, craving e consequência de vida negativa. Então, a partir desta, a MPPUSA foi adaptada e validada com 1.132 estudantes espanhóis 
de 12 a 18 anos, obtendo um alfa de Cronbach de 0,97 (Lopez-Fernandez et al., 2012). Também foi adaptada e validada para adolescentes britânicos, apresentando excelente consistência interna (alfa de Cronbach de 0,97) (Lopez-Fernandez et al., 2014).

Da mesma forma, uma versão resumida (MPPUS-10) foi adaptada partir da MPPUS, com apenas 10 itens e validada com 412 adolescentes suíços de 12 a 17 anos. $\mathrm{O}$ instrumento refletiu altamente o MPPUS original. A consistência interna foi boa, com o alfa de Cronbach 0,85 (Foerster et al., 2015).

Pesquisadores adaptaram a SAS para uma versão curta (SAS-SV). A SAS consiste em uma escala de 6 fatores e 33 itens em formato Likert de seis pontos com base no autorrelato: perturbação da vida diária, antecipação positiva, abstinência, relação orientada para o ciberespaço, uso excessivo e tolerância (Kwon et al., 2013a). Contudo, a SAS-SV adaptada conta com apenas 10 questões e foi aplicada em 599 adolescentes sul coreanos, alcançando uma boa consistência interna. A SAS-SV foi significativamente correlacionada com a escala SAS, SAPS e The Korean self-reporting Internet addiction scale short-form scale (KS) (Kwon et al., 2013a).

Outrossim, a SAPS foi desenvolvida e avaliada com 795 estudantes sul coreanos do ensino fundamental e médio. A escala final continha 15 itens no formato Likert, distribuídos em quatro subdomínios: perturbação de funções adaptativas, orientação de vida virtual, abstinência e tolerância. A escala final indicou alta confiabilidade $\operatorname{com} \alpha$ de Cronbach de 0,88 . A validade de critério foi demonstrada por sua relação com a escala de dependência de internet, KS-II $(r=0,49)$. Para a análise da validade de construto, foi testado o Modelo de Equações Estruturais, cujos resultados mostraram que a estrutura de quatro fatores é válida $(N F I=$ 0,94, $T L I=0,90, C F I=0,90, R M S E A=0,03)(\mathrm{Kim}$ et al., 2014).

Para determinar a capacidade preditiva dos traços de personalidade na DS, pesquisadores validaram o instrumento MPIQ, uma escala do tipo
Likert de oito itens que visa avaliar a associação cognitiva e comportamental dos participantes com seu telefone celular, com base em componentes da dependência comportamental. O índice de consistência interna foi avaliado em 242 adolescentes espanhóis e obteve um valor de 0,89 (Argumosa-Villar et al., 2017).

Ainda, o instrumento MPAI foi avaliado em 899 adolescentes chineses. Esta escala analisa quatro dimensões do vício em telefonia móvel com 17 itens, em formato tipo Likert, incluindo a incapacidade de controlar os desejos, ansiedade e sentimento de perda, abstinência e fuga, bem como a perda de produtividade. $\mathrm{O}$ alfa de Cronbach para o MPAI foi de 0,88 (Liu et al., 2017).

A escala KBUTK, composta por 33 itens, foi aplicada em 470 adolescentes poloneses e apresentou coeficientes de confiabilidade para as subescalas altos: escala de aceitação e proximidade Alfa de Cronbach $=0,88$, dependência de funções de uma escala de telefone celular $\alpha=0,75$, vício de enviar mensagens de texto e fazer chamadas telefônicas $\alpha=0,87$, dependência da escala de comunicação intermediária $\alpha=0,84$. O coeficiente de confiabilidade para a pontuação total do KBUTK foi $\alpha=0,93$ (Warzecha \& Pawlak, 2017).

Adicionalmente, uma pesquisa adaptou e validou a escala NMP-Q em 306 adolescentes espanhóis (González-Cabrera et al., 2017). O NMP-Q é uma escala Likert, com 20 itens, incluindo aspectos qualitativas e quantitativas. O NMP-Q abrange quatro fatores: incapacidade de se comunicar, perda de conexão, incapacidade de recuperar informações e abandono da conveniência. A escala adaptada obteve alfa de 0,95 , variando de 0,75 a 0,92 entre os fatores. A medida de estabilidade foi calculada pelo método teste e reteste $(r=0,82)$ (González-Cabrera et al., 2017). No Irã, uma versão persa do NMP-Q foi adaptada e validada com 3.216 adolescentes, alcançando similarmente uma consistência interna satisfatória $(\alpha=0,92)$ (Lin et al., 2018).

Em 2018, a associação entre o MPDQ e o Internet Addiction Test (IAT) foi investigada por meio do 
teste de validade concorrente com uma amostra de 1.072 adolescentes chineses. Os autores utilizaram o MPDQ desenvolvido inicialmente por Ezoe e colaboradores (2016), uma escala composta por 20 itens no formato Likert com o coeficiente de confiabilidade de 0,86 e confiabilidade ômega de 0,91 para a pontuação geral do MPDQ. Correlações mostraram que os escores das medidas de MPDQ e IAT foram moderadamente correlacionados, fornecendo mais evidências da validade de cada medida. Análises ROC mostraram taxas semelhantes de participantes com altas pontuações no IAT e no MPDQ (Chin \& Leung, 2018).

Por fim, o Implicit Association Test measures (IAT) com medidas para a dependência de smartphones e Internet (IAT-SI) foi desenvolvido e validado com 78 adolescentes sul coreanos, exibindo boa consistência interna $(\alpha=0,75)$, boa correlação significativa com a SAS-SV $(r=0,33, p<0,01)$ e boa validade convergente e discriminante do TAI-SI como uma nova medida relacionada à DS. O IAT-SI foi aplicado em formato informatizado usando o Inquisit 4.0 Millisecond Software (Roh et al., 2018).

\section{Discussão}

Esta revisão reuniu evidências científicas sobre as principais características da DS em adolescentes e os instrumentos de avaliação utilizados para rastrear o fenômeno. Identificou-se que, os artigos sobre DS em adolescentes foram publicados entre 2009 e 2019, com maior concentração nos últimos cinco anos. Certamente isso ocorreu devido a chegada ao mercado do smartphone com tela touch screen a partir de 2007, com uma interface mais complacente e melhor acesso à internet móvel, permitindo a conectividade com mobilidade aos seus usuários de maneira versátil e multifuncional (Birenboim \& Shoval, 2016).

Esse advento tornou o aparelho um símbolo de modernidade, gerando um impacto significativo na sociedade devido a sua presença constante junto aos usuários. Ao passo que, no final de 2014, mais de $93 \%$ da população mundial possuía telefones celulares, com taxas que ultrapassavam $100 \%$ na maioria dos países desenvolvidos (Birenboim \& Shoval, 2016), o que justifica o maior interesse nas pesquisas sobre DS a partir de então, reverberando em diversas áreas.

O uso anômalo desse dispositivo levou a questionamentos sobre a possibilidade de problemas e transtornos comportamentais vinculado a ele, sobretudo na Coreia do Sul, país conhecido pelos avanços no que diz respeito à adoção e uso de novas tecnologias de comunicação (Park et al., 2013), com alta penetração entre os indivíduos mais jovens (Gutiérrez et al., 2016).

A facilidade de acesso combinada com o uso generalizado e socialmente aceito de smartphones, torna o dispositivo indispensável. O crescente número de funcionalidades que o dispositivo oferece, torna os usuários mais dependentes da tecnologia e incentivam o uso do smartphone em relação a opções analógicas ou outros dispositivos digitais. Além disso, os aplicativos são projetados para fazer com que os usuários prolonguem seu uso ou voltem a eles, por exemplo, por meio de notificações ou recompensas diárias pedindo ao usuário para abrir o aplicativo (Noë et al., 2019).

Nesta revisão não foram encontrados estudos desenvolvidos nos países do Continente Americano, como o Brasil, embora o smartphone seja o equipamento mais utilizado no país para o acesso à internet e está presente em praticamente todos os lares brasileiros (IBGE, 2020).

Entre os estudos analisados nesta revisão, verificou-se que a maioria era do tipo transversal, seguido pelos metodológicos, os quais contribuem para existência de instrumentos adaptados e válidos para mensuração da condição na população adolescente, bem como do conhecimento sobre a sua prevalência e fatores associados ao fenômeno, abordados na parte 2 desta revisão (Freitas et al., 2021).

Por tratar-se de um novo construto multidimensional na área de saúde, faz-se necessário a elaboração 
e validação de instrumentos que sejam capazes de mensurar o fenômeno em questão com boas propriedades psicométricas. Por isso, esse tipo de estudo é tão necessário (Souza et al., 2017). Contudo, como estas avaliações são de autorrelato, apresentam um desempenho limitado e, por isso, os pesquisadores devem ser cautelosos ao usar estas medidas para apoiar diagnósticos. Portanto, pouco se sabe sobre a estabilidade da condição, e não está claro se os sintomas autorrelatados podem ser validados usando métodos diagnósticos mais formais (Ellis et al., 2019).

Outra revisão sobre DS também verificou que a maioria dos estudos sobre o tema é transversal e baseia-se em questionários aplicados a estudantes em amostras de conveniência que normalmente contêm apenas um ponto de amostragem, embora vários estudos recentes tenham sido baseados em registros telefônicos longitudinais (Gutiérrez et al., 2016).

As características da DS descritas nesta revisão são semelhantes as apresentadas em outras pesquisas (Gutiérrez et al., 2016; Khoury et al., 2017), destacando-se principalmente os sintomas de abstinência (ansiedade, irritabilidade e impaciência) (Körmendi et al., 2016), falta de controle no uso, tempo de uso mais longo do que o inicialmente previsto (Beranuy et al., 2009), sintomas de tolerância (Körmendi et al., 2016), interferências nas atividades de vida diária (Beranuy et al., 2009), antecipação positiva e manutenção do tempo de uso apesar de consequências negativas (Kwon et al., 2013b).

Pesquisa realizada no Brasil com 100 estudantes de pós-graduação, com idade entre 18 e 25 anos, identificou que dependentes de smartphones apresentaram comprometimento na tomada de decisão sob ambiguidade com preservação de tomada de decisão sob risco, portanto eles tendem a preferir alternativas vantajosas no curto prazo, mesmo quando trazem maiores perdas futuras. Esses indivíduos apresentaram maior sensibilidade a recompensas e menor sensibilidade a punições. Portanto, quando uma alternativa gera alta recompensa, ela é escolhida mesmo que gere maior punição a longo prazo. Este perfil de "miopia para o futuro" em momentos decisivos pode contribuir para a iniciação e manutenção de comportamentos aditivos (Khoury et al., 2019).

O público adolescente é estereotipado por seu envolvimento em comportamentos de risco (Blakemore \& Mills, 2014). Evidencia-se que há atividade intensificada no sistema de recompensa dopaminérgica mesolímbica, o que apoia a hipótese de que os adolescentes são propensos a assumirem riscos devido a circuitos hiperativos relacionados à recompensa. Portanto, mudanças nos comportamentos e preferências de busca por recompensa são impulsionadas, em parte, por mudanças neurais subjacentes no circuito frontostriatal. Esta atividade neural é significativamente maior entre adolescentes do que crianças ou adultos ao receber recompensas secundárias, o que os expõem a um maior risco de desenvolverem a DS (Telzer, 2016).

Portanto, a maior sensibilidade a "curtidas" e "comentários" nas redes sociais, por exemplo, pode ser um fator de vulnerabilidade para o desenvolvimento da DS, mesmo quando há perdas ou possibilidade de danos em diversas áreas da vida, como a redução do desempenho acadêmico, os acidentes de trânsito e os prejuízos nas relações interpessoais (Khoury et al., 2019).

Além disso, a influência de estímulos externos (como a vibração do smartphone, emissão de sons e luzes, ou mesmo a visualização de pessoas utilizando o dispositivo) pode contribuir para a hiperativação do sistema impulsivo, e aumento no uso do smartphone, consequentemente. Tal condição pode cooperar para a redução da funcionalidade e para a geração de sofrimento para si e para outros, características essenciais para considerá-la como um agravo psiquiátrico (Khoury et al., 2019).

Por meio desta revisão foi possível identificar 14 escalas validadas para avaliação da dependência autorreferida de smartphone em adolescentes, sendo que, as mais utilizadas pelos estudos analisados foram a SAPS e a SAS-SV. No entanto, estudo anterior apontou que a SAS e o Smartphone Addiction Inventory (SPAI) eram os instrumentos de rastreio 
mais utilizados pelas pesquisas desenvolvidas com a população geral (Khoury et al., 2017). Ressalta-se que não foram identificados estudos de adaptação e validação da SPAI para a população adolescente nesta revisão, considerando o período de buscas divulgado.

Em geral, esses instrumentos evoluíram do estudo do comportamento global do uso de telefones celulares para comportamentos específicos, como o uso de smartphones (Gutiérrez et al., 2016). Analisando de forma geral os diferentes instrumentos, verifica-se que existem aspectos comuns na avaliação, a destacar os sintomas: abstinência, tolerância, compulsão e efeito na vida diária. Embora exista um consenso sobre a existência da DS, a sua delimitação e os critérios utilizados por vários pesquisadores variam (Gutiérrez et al., 2016). A consistência interna das escalas incluídas nesta revisão variou de 0,80 a 0,97 , considerada satisfatória para medir o respectivo construto e reproduzir um resultado de forma consistente no tempo e no espaço (Souza et al., 2017).

O maior obstáculo à pesquisa sobre a DS é a diversidade de termos, critérios e definições/conceitos disponíveis neste campo, como o "uso problemático", "abuso" e "adição/dependência". Isso pode explicar o grande volume de instrumentos e a diferença entre os domínios que os compõem, dificultando a comparação dos achados (Gutiérrez et al., 2016).

Diante do que foi encontrado e discutido até o momento, pode-se considerar que os instrumentos que estão sendo desenvolvidos e validados para avaliar a dependência autorreferida por adolescentes foram utilizados em uma variedade de contextos. Na maioria das vezes eles não foram aplicados de forma isolada, pois sempre havia outras variáveis de avaliação envolvidas, como a qualidade de vida, desempenho escolar, sintomas depressivos e neuropsiquiátricos, dentre outros, além da presença de outros testes e questionários que avaliavam tais variáveis. Isto reflete a complexidade conceitual da DS, envolvendo diversos aspectos da saúde dos indivíduos.
Embora não tenha sido identificado por esta revisão nenhum estudo que versasse sobre instrumento de avaliação da DS adaptado e validado com adolescentes brasileiros, recentemente, o instrumento SPAI foi traduzido, adaptado e validado para a população jovem brasileira de 18 a 35 anos, sendo denominado SPAI-BR (Khoury et al., 2017). Os autores optaram por validar o questionário SPAI, pois é mais curto e mais fácil de responder, portanto, mais adequado para ser utilizado no sistema público de saúde brasileiro. Esta versão manteve as equivalências semântica, idiomática e conceitual da escala original, com boa consistência interna $(0,88)$ e excelente estabilidade $(0,92)$.

A escala foi considerada uma ferramenta válida e confiável para o rastreamento da dependência de smartphone em jovens brasileiros numa versão dicotômica (sim e não), visando à redução do tempo de preenchimento (Khoury et al., 2017). A SPAI original possui 26 itens no formato Likert e avalia quatro fatores: comportamento compulsivo, comprometimento funcional, síndrome de abstinência e síndrome de tolerância (Lin et al., 2014).

Além disso tem-se a Escala de dependência de smartphone (EDS), adaptada e validada com indivíduos brasileiros de 15 a 53 anos. Este instrumento foi adaptado a partir da Escala de Uso Compulsivo da Internet (CIUS). Após a sua adaptação e validação, manteve-se o formato Likert, com 14 itens e um bom índice de consistência interna com alfa de 0,94 . Os autores justificam que há uma similaridade entre a Internet e o smartphone, já que esse último segue os mesmos princípios do primeiro considerando suas inúmeras aplicações, que abarcam inclusive o acesso à Internet (Sales et al., 2018).

Frente a complexidade da DS, nota-se a importância do caráter interdisciplinar e interprofissional no trato dos fatores de proteção dos adolescentes na era digital, aliando e harmonizando os setores da família, educação e saúde para garantir o direito de proteção integral. Os benefícios e prejuízos dessas tecnologias permanecem sendo foco de atenção de todos os profissionais que lidam com as questões 
da saúde durante a adolescência e demandam novos conhecimentos a fim de subsidiar suas práticas (Sociedade Brasileira de Pediatria [SBP], 2016).

Por limitar idiomas e base de dados, esta revisão pode ter restringido o acesso a outros dados relevantes já publicados. Espera-se que a partir deste estudo possam surgir outras pesquisas brasileiras, apresentando contribuições no que tange a temática, na adaptação e validação de instrumentos, na identificação de fatores de risco e de proteção e subsidiando a reformulação e reestruturação de práticas e políticas relacionadas ao uso não prejudicial do smartphone pelos adolescentes.

\section{Conclusão}

Conclui-se que a DS é um problema de saúde pública em ascensão, que está intimamente ligada ao desenvolvimento tecnológico e pode comprometer a saúde dos adolescentes. Os estudos encontrados no período da busca foram, em sua maioria, transversais e metodológicos, carecendo de pesquisas com metodologias mais robustas para melhor compreensão do fenômeno.

O maior obstáculo à pesquisa sobre a DS é a diversidade de termos, ausência de uma definição consensual de critérios e classificações disponíveis neste campo. Isso pode explicar o grande volume de instrumentos e a diferença entre os domínios que os compõem, dificultando a comparação dos achados. Foram identificadas 14 escalas validadas e confiáveis para avaliação da DS em adolescentes, que contemplam, em sua maioria, os fatores abstinência, tolerância, compulsão e efeitos na vida diária.

Porém, no período analisado não foram identificados estudos brasileiros, portanto, faz-se necessário investir em estudos de adaptação e validação de escalas já utilizadas em outros contextos para a população de adolescentes brasileiros, pois as pesquisas dessa área perdem valor e confiabilidade quando não utilizam instrumentos apropriados para a própria realidade.
Reforça-se a importância de sensibilizar os profissionais de saúde sobre a temática que, apesar de ainda pouco estudada, aponta para um importante problema de saúde pública atual. Espera-se que os resultados obtidos neste estudo possam ilustrar a necessidade de unificação do conceito de DS, bem como da importância da delimitação de instrumentos específicos para sua avaliação em adolescentes brasileiros a fim de possibilitar novas pesquisas neste campo à luz da limitação de seus conceitos, critérios, metodologias e localidades.

Além do mais, é importante possibilitar aos profissionais de saúde o rastreio de tal condição durante a vigilância em saúde do adolescente no âmbito do cuidado primário em saúde, para que haja melhor planejamento e implementação de medidas preventivas deste comportamento de risco e protetivas, em prol de comportamentos adaptativos que garantam o desenvolvimento pleno desse grupo populacional.

\section{Referências}

American Psychiatric Association. (2014). Manual diagnóstico e estatístico de transtornos mentais- DSM-5 (5 ed.). American Psychiatric Association.

Antunes, J. L. F., \& Cardoso, M. R. A. (2015). Uso da análise de séries temporais em estudos epidemiológicos. Epidemiologia e Serviços de Saúde, 24(3), 565-576. https://doi.org/10.5123/ S1679-49742015000300024

Argumosa-Villar, L., Boada-Grau, J., \& Vigil-Colet, A. (2017). Exploratory investigation of theoretical predictors of nomophobia using the Mobile Phone Involvement Questionnaire (MPIQ). Journal of Adolescence, 56, 127-135. https:// doi.org/10.1016/j.adolescence.2017.02.003

Beranuy F. M., Chamarro L. A., Graner J. C., \& Carbonell, X. (2009). Validación de dos escalas breves para evaluar la adicción a internet y el abuso de móvil. Psicothema, 21(3), 480-485. https:// www.redalyc.org/pdf/727/72711821023.pdf 
Birenboim, A., \& Shoval, N. (2016). Mobility research in the age of the smartphone. Annals of the American Association of Geographers, 106(2), 283-291. https://doi.org/10.1080/000 45608.2015.1100058

Blakemore, S.J., \& Mills, K. L. (2014). Is Adolescence a sensitive period for sociocultural processing? Annual Review of Psychology, 65(1), 187-207. https://doi.org/10.1146/annurev-psych-010213-115202

Blanco, M. de G., Anglada, M. V., Pérez, M. J. F., $\&$ Arbonès, M. M. (2002). Problemas conductuales relacionados con el uso de Internet: un estudio exploratorio. Anales de Psicología, 18(2), 273-292. https://revistas.um.es/analesps/ article/view/28491

Chin, F., \& Leung, C. H. (2018). The concurrent validity of the Internet addiction test (IAT) and the mobile phone dependence questionnaire (MPDQ). PLOS ONE, 13(6), Artigo e0197562. https://doi. org/10.1371/journal.pone.0197562

Chóliz, M. (2012). Mobile-phone addiction in adolescence: The test of mobile phone dependence (TMD). Progess in Health Sciences, 2(1), 33-44. https://acortar.link/6Kwm2W

Chun, J. W., Choi, J., Cho, H., Choi, M. R., Ahn, K. J., Choi, J. S., \& Kim, D. J. (2018). Role of frontostriatal connectivity in adolescents with excessive smartphone use. Frontiers in Psychiatry, 9, Artigo 437. https://doi.org/10.3389/ fpsyt.2018.00437

CISCO (2020). Cisco Annual Internet Report (20182023). CISCO - Computer Fraud \& Security. https://doi.org/10.1016/s1361-3723(20)30026-9.

Cocoradă, E., Maican, C. I., Cazan, A. M., \& Maican, M. A. (2018). Assessing the smartphone addiction risk and its associations with personality traits among adolescents. Children and Youth Services Review, 93, 345-354. https://doi. org/10.1016/j.childyouth.2018.08.006

Coluci, M. Z. O., Alexandre, N. M. C., \& Milani, D. (2015). Construção de instrumentos de medida na área da saúde. Ciencia e Saude Coletiva, 20(3), 925-936. https://doi.org/10.1590/141381232015203.04332013

Duke, É., \& Montag, C. (2017). Smartphone addiction, daily interruptions and self-reported productivity. Addictive Behaviors Reports, 6, 90-95. https:// doi.org/10.1016/j.abrep.2017.07.002

Ellis, D. A., Davidson, B. I., Shaw, H., \& Geyer, K. (2019). Do smartphone usage scales predict behavior? International Journal of Human Computer Studies, 130, 86-92. https://doi.org/10.1016/j. ijhcs.2019.05.004

Ezoe, S., Iida, T., Inoue, K., \& Toda, M. (2016). Development of Japanese Version of Smartphone Dependence Scale. Open Journal of Preventive Medicine, 06(07), 179-185. https://doi. org/10.4236/ojpm.2016.67017

Freitas, B. H. B. M., Gaíva, M. A. M., Bernardino, F. B. S., \& Diogo, P. M. J. (2021). Smartphone Addiction in Adolescents, part 2: Scoping Review-Prevalence and Associated Factors. Trends in Psychology, 29, 12-30. https://doi. org/10.1007/s43076-020-00040-4

Foerster, M., Roser, K., Schoeni, A., \& Röösli, M. (2015). Problematic mobile phone use in adolescents: Derivation of a short scale MPPUS-10. International Journal of Public Health, 60(2), 277-286. https://doi.org/10.1007/s00038-0150660-4

González-Cabrera, J., León-Mejía, A., Pérez-Sancho, C., \& Calvete, E. (2017). Adaptation of the Nomophobia Questionnaire (NMP-Q) to Spanish in a sample of adolescents. Actas Espanolas de Psiquiatría, 45(4), 137-144. https://europepmc. org/article/med/28745386

Goodman, A. (1990). Addiction: Definition and implications. British Journal of Addiction, 85(11), 14031408. https://doi.org/10.1111/j.1360-0443.1990. tb01620.x

Griffiths, M. (1996). Gambling on the Internet: A Brief Note. Journal of Gambling Studies, 12(4), 471-473. https://acortar.link/QGAPgB 
Griffiths, M. (2000). Does Internet and Computer "Addiction" Exist? Some Case Study Evidence. CyberPsychology \& Behavior, 3(2), 211-218. https://doi.org/10.1089/109493100316067

Gutiérrez, J. D. S., De Fonseca, F. R., \& Rubio, G. (2016). Cell-phone addiction: A review. Frontiers in Psychiatry, 7, Artigo 715. https://doi. org/10.3389/fpsyt.2016.00175

Güzeller, C. O., \& Coşguner, T. (2012). Development of a problematic mobile phone use scale for Turkish adolescents. Cyberpsychology, Behavior, and Social Networking, 15(4), 205-211. https://doi.org/10.1089/cyber.2011.0210

Instituto Brasileiro de Geografia e Estatística [IBGE]. (2020). Pesquisa Nacional por Amostra de Domicílios. Acesso à Internet e à televisão e posse de telefone móvel celular para uso pessoal. IBGE. https://biblioteca.ibge.gov.br/ visualizacao/livros/liv101705_informativo.pdf

Jeong, S. H., Kim, H. J., Yum, J. Y., \& Hwang, Y. (2016). What type of content are smartphone users addicted to?: SNS vs. games. Computers in Human Behavior, 54, 10-17. https://doi.org/10.1016/j.chb.2015.07.035

Khoury, J. M., Couto, L. F. S. C., Santos, D. de A., e Silva, V. H. de O., Drumond, J. P. S., Silva, L. L. de C. e, Malloy-Diniz, L., Albuquerque, M. R., das Neves, M. de C. L., \& Duarte Garcia, F. (2019). Bad Choices Make Good Stories: The Impaired Decision-Making Process and Skin Conductance Response in Subjects With Smartphone Addiction. Frontiers in Psychiatry, 10, Artigo 73. https://doi. org/10.3389/fpsyt.2019.00073

Khoury, J. M., De Freitas, A. A. C., Roque, M. A. V., Albuquerque, M. R., Das Neves, M. D. C. L., \& Garcia, F. D. (2017). Assessment of the accuracy of a new tool for the screening of smartphone addiction. PLOS ONE, 12(5), Artigo e0176924. https://doi.org/10.1371/journal.pone.0176924

Kim, D., Lee, Y., Lee, J., Nam, J. E. K., \& Chung, Y. (2014). Development of Korean Smartphone Ad- diction Proneness Scale for youth. PLOS ONE, 9(5), Artigo e97920. https://doi.org/10.1371/journal. pone. 0097920

Körmendi, A., Brutóczki, Z., Végh, B. P., \& Székely, R. (2016). Smartphone use can be addictive? A case report. Journal of Behavioral Addictions, 5(3), 548-552. https://doi.org/10.1556/2006.5.2016.033

Kwon, M., Kim, D. J., Cho, H., \& Yang, S. (2013a). The smartphone addiction scale: Development and validation of a short version for adolescents. PLOS ONE, 8(12), Artigo e83558. https://doi. org/10.1371/journal.pone.0083558

Kwon, M., Lee, J.-Y., Won, W.-Y., Park, J.-W., Min, J.-A., Hahn, C., Gu, X., Choi, J.-H., \& Kim, D.-J. (2013b). Development and Validation of a Smartphone Addiction Scale (SAS). PLOS ONE, 8(2), Artigo e56936. https://doi.org/10.1371/ journal.pone.0056936

Lin, C.Y., Griffiths, M. D., \& Pakpour, A. H. (2018). Psychometric evaluation of Persian Nomophobia Questionnaire: Differential item functioning and measurement invariance across gender. Journal of Behavioral Addictions, 7(1), 100108. https://doi.org/10.1556/2006.7.2018.11

Lin, Y. H., Chang, L. R., Lee, Y. H., Tseng, H. W., Kuo, T. B. J., \& Chen, S. H. (2014). Development and validation of the Smartphone Addiction Inventory (SPAI). PLOS ONE, 9(6), Artigo e98312. https://doi.org/10.1371/journal.pone.0098312

Liu, Q. Q., Zhou, Z. K., Yang, X. J., Kong, F. C., Niu, G. F., \& Fan, C. Y. (2017). Mobile phone addiction and sleep quality among Chinese adolescents: A moderated mediation model. Computers in Human Behavior, 72, 108-114. https://doi.org/10.1016/j.chb.2017.02.042

Lopez-Fernandez, O., Honrubia-Serrano, M. L., \& Freixa-Blanxart, M. (2012). Spanish adaptation of the "Mobile Phone Problem Use Scale" for adolescent population. Adicciones, 24(2), 123-130. https://www.proquest.com/openview/ c7f9d09fde010d8687f97857abdbe0b5/1?pq-origsite $=$ gscholar $\&$ cbl $=2032270$ 
Lopez-Fernandez, O., Honrubia-Serrano, M. L., Freixa-Blanxart, M., \& Gibson, W. (2014). Prevalence of Problematic Mobile Phone Use in British Adolescents. Cyberpsychology, Behavior, and Social Networking, 17(2), 91-98. https://doi.org/10.1089/ cyber.2012.0260

Noë, B., Turner, L. D., Linden, D. E. J., Allen, S. M., Winkens, B., \& Whitaker, R. M. (2019). Identifying Indicators of Smartphone Addiction Through User-App Interaction. Computers in Human Behavior, 99, 56-65. https://doi.org/10.1016/j.chb.2019.04.023

Page, M. J., McKenzie, J. E., Bossuyt, P. M., Boutron, I., Hoffmann, T. C., Mulrow, C. D., Shamseer, L., Tetzlaff, J. M., Akl, E. A., Brennan, S. E., Chou, R., Glanville, J., Grimshaw, J. M., Hróbjartsson, A., Lalu, M. M., Li, T., Loder, E. W., Mayo-Wilson, E., McDonald, S., ... Moher, D. (2021). The PRISMA 2020 statement: An updated guideline for reporting systematic reviews. $B M J, 372$, Artigo n71. https://doi.org/10.1136/ bmj.n71

Park, N., Kim, Y., Young, H., \& Shim, H. (2013). Factors influencing smartphone use and dependency in South Korea. Computers in Human Behavior, 29(4), 1763-1770. https://doi. org/10.1016/j.chb.2013.02.008

Sociedade Brasileira de Pediatria [SBP]. (2016, octubre). Saúde de Crianças e Adolescentes na Era Digital. In SBP (Org.), Manual de orientação: Departamento de adolescência (N. 1, pp. 1-13). https://www.sbp.com.br/fileadmin/ user upload/2016/11/19166d-MOrient-Saude-Crian-e-Adolesc.pdf

Peters, M. D. J., Godfrey, C., McInerney, P., Munn, Z., Tricco, A. C., \& Khalil, H. (2020). Chapter 11: Scoping Reviews. In E. Aromataris \& Z. Munn (Eds.), JBI Reviewer's Manual. https:// doi.org/10.46658/JBIMES-20-12
Roh, D., Bhang, S.-Y., Choi, J.-S., Kweon, Y. S., Lee, S.-K., \& Potenza, M. N. (2018). The validation of Implicit Association Test measures for smartphone and Internet addiction in at-risk children and adolescents. Journal of Behavioral Addictions, 7(1), 79-87. https://doi. org/10.1556/2006.7.2018.02

Ruiz, J., Sánchez, J., \& Trujillo, J. (2016). Utilizacion de Internet y dependencia a teléfonos móviles en adolescentes. Revista Latinoamericana de Ciencias Sociales, Niñez y Juventud, 14(2), 1357-1369. https://doi.org/10.11600/169271 5x.14232080715

Sales, H. F. S., Silva, F. M. de S. M. da, Lopes, B. de J., \& Lima, C. F. de. (2018). Adaptação da escala de uso compulsivo de Internet para avaliar dependência de smartphone. Avances en Psicologia Latinoamericana, 36(1), 155-166. https://doi.org/10.12804/revistas.urosario.edu. co/apl/a.4649

Santana-Vega, L.-E., Gómez-Muñoz, A.-M., \& Feliciano-garcía, L. (2019). Adolescents problematic mobile phone use, fear of missing out and family communication. Comunicar Journal, 27(59), 39-47. https://doi.org/10.3916/C592019-04

Silva, T. D. O., \& Silva, L. T. G. (2017). Os impactos sociais, cognitivos e afetivos sobre a geração de adolescentes conectados às tecnologias digitais. Revista Psicopedagogia, 34(103), 87-97. http://pepsic.bvsalud.org/pdf/psicoped/ v34n103/09.pdf

Souza, A. C. de, Alexandre, N. M. C., \& Guirardello, E. de B. (2017). Propriedades psicométricas na avaliação de instrumentos: avaliação da confiabilidade e da validade. Epidemiologia e Serviços de Saúde: Revista do Sistema Único de Saúde do Brasil, 26(3), 649-659. https:// doi.org/10.5123/S1679-49742017000300022 
Telzer, E. H. (2016). Dopaminergic reward sensitivity can promote adolescent health: A new perspective on the mechanism of ventral striatum activation. Developmental Cognitive Neuroscience, 17, 57-67. https://doi.org/10.1016/j. den.2015.10.010

Warzecha, K., \& Pawlak, A. (2017). Pathological use of mobile phones by secondary school students. Archives of Psychiatry and Psychotherapy,
19(1), 27-36. https://doi.org/10.12740/app/ 67931

Yen, C. F., Tang, T. C., Yen, J. Y., Lin, H. C., Huang, C. F., Liu, S. C., \& Ko, C. H. (2009). Symptoms of problematic cellular phone use, functional impairment and its association with depression among adolescents in Southern Taiwan. Journal of Adolescence, 32(4), 863-873. https://doi. org/10.1016/j.adolescence.2008.10.006 